\title{
Cryptococcosis in HIV negative patients: findings on chest radiography
}

\author{
Derek J Roebuck, Dale A Fisher, Bart J Currie
}

\begin{abstract}
Background-The findings at chest radiography in HIV negative patients with cryptococcosis are said to differ from those in HIV positive patients. No large series of HIV negative patients has been reported.

Methods-A review of the medical charts and radiographs of 44 consecutive HIV negative patients with proven cryptococcosis was undertaken.

Results-Air space consolidation involving one or more lobes was found in 15 patients $(34 \%)$, one or more pulmonary masses in 13 patients $(30 \%)$, and interstitial opacities in three patients $(7 \%)$. No other radiographic finding was detected in more than one patient. Twelve of 23 patients $(52 \%)$ with cryptococcal meningitis had abnormal chest radiographs, and 10 of 16 patients $(62 \%)$ who presented with respiratory symptoms had air space consolidation.

Conclusions-HIV negative patients tend to have different radiographic findings from those reported in patients with AIDS. Cryptococcal meningitis in this group is usually associated with pulmonary mass(es) or a normal chest radiograph, whereas a respiratory presentation is usually associated with air space consolidation.

(Thorax 1998;53:554-557)
\end{abstract}

Keywords: cryptococcosis; diagnosis; radiography

Cryptococcus neoformans is a ubiquitous saprophytic fungus with a characteristic polysaccharide capsule. Two varieties, $C$ neoformans var neoformans and $C$ neoformans var gattii, were first recognised on morphological grounds ${ }^{1}$ and this distinction is supported by genetic, serological, epidemiological, and clinical $^{2}$ evidence. Inoculation is usually by inhalation, with both varieties potentially causing disease in humans. Pulmonary involvement may be followed by haematogenous spread to the central nervous system or, less commonly, to other organs such as bone and skin.

Three main patterns have been described on chest radiography in immunocompetent individuals ${ }^{3-6}$ : solitary or multiple masses of more than $5 \mathrm{~mm}$ in diameter and often much larger; patchy, segmental or lobar air space consolidation which is often multifocal; and nodular or reticulonodular interstitial changes. Immunosuppression appears to affect the pattern of pulmonary involvement. ${ }^{3}$ In patients with AIDS interstitial changes are common and are often accompanied by lymphadenopathy. ${ }^{7}$

The tropical "top end" of the Northern Territory of Australia has the highest documented incidence of cryptococcosis in the world. Aboriginal Australians, in the absence of AIDS, are 20 times more likely to become infected than non-aboriginals. ${ }^{8}$

We present an analysis of the chest radiographs of $44 \mathrm{HIV}$ negative patients with cryptococcosis who were treated at a single hospital between 1976 and 1997.

\section{Methods}

The institution is the only general hospital in a region of over $520000 \mathrm{~km}^{2}$. It serves a population of 130000 of whom $22 \%$ are aboriginal. Patients diagnosed with cryptococcosis from 1976 to 1997 were identified by a search of medical charts, microbiology records, and data kept by the Department of Medicine. This study is a review of the chest radiographs of these patients and their clinical correlations. Clinical data collected included age, sex, ethnicity, clinical features, underlying diseases, and diagnostic criteria. The clinical features, management, and outcome of some of these patients have been previously reported. ${ }^{8}$

Forty six patients were identified, two of whom were HIV antibody positive and were excluded, leaving 44 patients for analysis. Testing for HIV antibody has been routine in these patients for the last decade and it is assumed that all earlier patients were HIV antibody negative.

The diagnosis of cryptococcosis was made if clinical criteria were met and supported by identification of $C$ neoformans in blood, sputum, cerebrospinal fluid or tissue using culture, histological examination or antigen detection. The findings on the frontal and lateral chest radiographs obtained on admission in all patients were classified using the following definitions, modified from the recommendations of the Fleischner Society. ${ }^{9}$

A mass was defined as any pulmonary lesion represented by a discrete opacity with a diameter of $5 \mathrm{~mm}$ or more, visible in both posteroanterior and lateral films. Air space opacification was defined as an essentially homogeneous opacity characterised by effacement of blood vessel shadows and little or no loss of volume; air bronchograms were sometimes present. Interstitial opacities were defined as a collection of small linear and/or micronodular opacities assumed to represent disease of the interstitium of the lung. Any other pertinent radiographic findings were also recorded. 
Table 1 Chest radiograph patterns in 44 HIV negative patients with cryptococcosis

\begin{tabular}{lccclc}
\hline Presentation & $\begin{array}{l}\text { Normal chest } \\
\text { radiograph }\end{array}$ & $\begin{array}{l}\text { Air space } \\
\text { consolidation }\end{array}$ & Mass(es) & $\begin{array}{l}\text { Interstitial } \\
\text { opacities }\end{array}$ & Total \\
\hline Meningitis & 11 & 4 & 8 & 0 & 23 \\
Respiratory & 0 & 10 & 3 & 3 & 16 \\
Fungaemia & 1 & 1 & 0 & 0 & 2 \\
Skin infection & 1 & 0 & 0 & 0 & 1 \\
Asymptomatic & 0 & 0 & 2 & 0 & 2 \\
Total & 13 & 15 & 13 & 3 & 44 \\
Immunocompetent & 6 & 8 & 8 & 0 & 22 \\
Possibly & 7 & 7 & 5 & 3 & 22 \\
$\quad$ immunosuppressed & & & & & \\
\hline
\end{tabular}

\section{Results}

The study included 44 patients (27 men) of median age 34 years (range 1-76), 39 of whom were aboriginal Australians. Twenty three presented with meningitis, 16 with a respiratory illness, two with fungaemia with no known primary source, and one with cutaneous infection. Two patients were asymptomatic and had abnormalities detected on chest radiographs performed for other reasons.

Twenty two of the patients, including 10 of $16(62 \%)$ with respiratory illness and nine of $23(39 \%)$ with meningitis, had conditions which may have predisposed them to cryptococcal infection. These included alcohol abuse $(n=6)$, chronic lung disease $(n=4)$, malnutrition $(\mathrm{n}=2)$, pulmonary tuberculosis $(\mathrm{n}=2)$, pregnancy $(n=2)$, syphilis with or without leprosy $(n=2)$, diabetes mellitus and splenectomy ( $\mathrm{n}=1)$, systemic lupus erythematosus with corticosteroid therapy $(n=1)$, chronic lung disease and cirrhosis $(n=1)$, and liver failure $(n=1)$.

The findings at chest radiography are summarised in tables 1 and 2. The radiographs of 13 patients showed a pulmonary mass (fig 1 ), including one patient who had two masses and two who had three. No calcification or cavitation was evident in these masses. Air space consolidation, usually patchy but occasionally segmental or lobar (fig 2), was seen in 15 patients, often involving more than one lobe. Cavitation was observed in only one of these patients. Interstitial abnormalities, either reticular or reticulonodular, were observed in three patients. The distinction between air space and interstitial patterns was difficult in some patients whose radiographs were only mildly abnormal. None of the 44 patients had both a mass and one of the other patterns. Twelve (52\%) of the 23 patients who presented with cryptococcal meningitis had abnormal chest radiographs, including eight patients with a pulmonary mass or masses (table 1). Ten $(62 \%)$ of the 16 patients with respiratory symptoms had air space opacification.

Table 2 Lobar distribution of radiographic abnormalities in 31 patients with cryptococcosis and abnormal chest radiographs (values in columns refer to numbers of lobes involved)

\begin{tabular}{lclclc}
\hline Radiographic pattern & RUL & RML & RLL & LUL & LLL \\
\hline Air space consolidation (n=15) & 6 & 6 & 8 & 5 & 6 \\
Mass(es) (n=13) & 4 & 1 & 8 & 0 & 5 \\
Interstitial opacities (n=3) & 0 & 0 & 2 & 0 & 3 \\
Total (n=31 patients) & 10 & 7 & 18 & 5 & 14
\end{tabular}

RUL = right upper lobe; $\mathrm{RML}=$ right middle lobe; $\mathrm{RLL}=$ right lower lobe; $\mathrm{LUL}=$ left upper lobe; LLL $=$ left lower lobe.

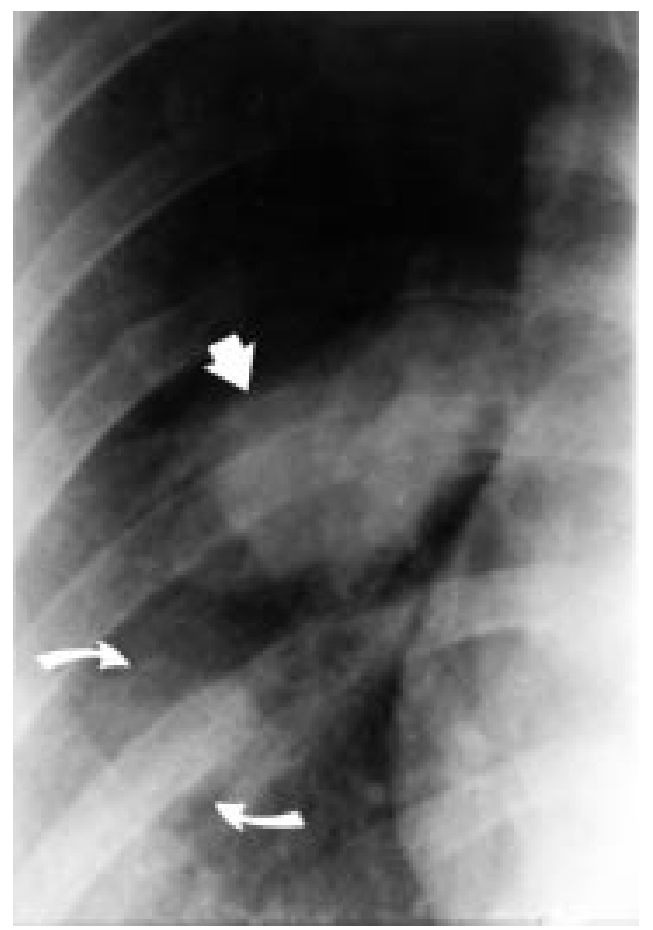

Figure 1 Chest radiograph of 28 year old immunocompetent aboriginal woman with cryptococcal meningitis ( $C$ neoformans var gattii) showing a large mass near the right hilum (arrow) and a second smaller mass lying peripherally (curved arrows).

Less common findings were observed in one patient each: pleural effusion, apparent cyst formation within an area of interstitial change, and hilar node calcification in a patient with previous pulmonary tuberculosis. Lesions which appeared to be a mass in one projection and an infiltrate in the orthogonal projection, described by Feigin ${ }^{10}$ as "infiltrative masses", were specifically sought but were not observed in any of these cases.

Masses occurred more often in the right lung ( $n=13)$ than the left $(n=5)$, and in the lower lobes $(n=13)$ more often than the upper and middle $(n=5)$. Neither of these differences was statistically significant (two tailed binomial distribution, $\mathrm{p}=0.096$ ). Air space consolidation showed no lobar predilection, and interstitial

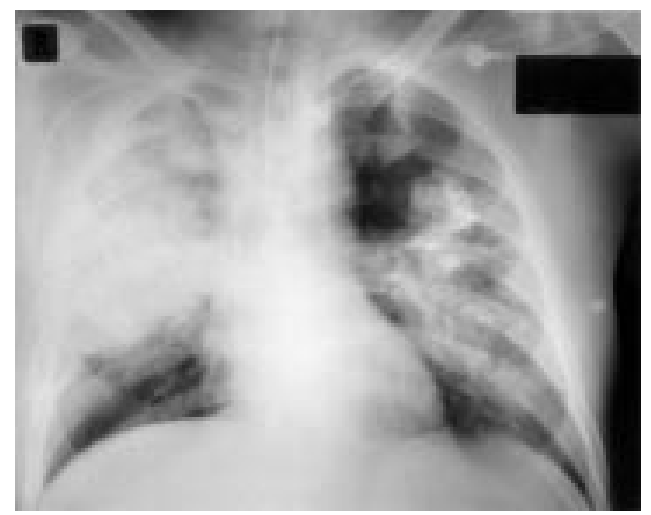

Figure 2 Chest radiograph of a 61 year old aboriginal man with diabetes mellitus and previous splenectomy for trauma who presented with cryptococcal pneumonia ( $C$ neoformans var gattii) showing extensive bilateral air space consolidation. A few faint air bronchograms were visible in the right upper lobe on the original film. 
opacity was observed in three patients, all with lower lobe involvement (table 2).

Variety identification was performed in 30 patients, of whom 24 showed $C$ neoformans var gattii and six showed $C$ neoformans var neoformans. $C$ neoformans var neoformans infection caused meningitis in two patients (normal chest radiographs), a respiratory illness in two patients (air space opacification), and fungaemia in two patients (one with normal radiographs and one with widespread air space opacification).

\section{Discussion}

Most recent reviews of the pulmonary features of cryptococcosis have involved patients with AIDS. ${ }^{6}{ }^{11} 12$ Since early in the AIDS epidemic it has been believed that the radiographic findings are different in this group of immunocompromised patients ${ }^{3}$ and, in particular, that pulmonary masses are uncommon. ${ }^{7}$ An interstitial lung pattern and lymphadenopathy appear to be more common in AIDS patients than in immunocompetent individuals. ${ }^{71}$ This probably reflects a difference in host response to cryptococcal infection in patients with defective $\mathrm{T}$ lymphocyte function. ${ }^{7}$ The tendency of AIDS patients to have infection with $C$ neoformans var neoformans instead of $C$ neoformans var gattii may also contribute to the differing radiological appearances. An alternative explanation is that other infective and non-infective complications of AIDS may be producing some of these radiographic signs.

Distinction between interstitial and air space patterns can be difficult, not least because most diseases of the pulmonary acinus which cause radiographic opacity involve both the air space and interstitial tissue. ${ }^{13}$ There is also likely to be intra- and inter-observer variation in interpretation. It may be difficult to distinguish between an area of air space consolidation and a mass, particularly since the definition of a mass used in this study specifically excludes assessment of contour and border characteristics. ${ }^{9}$ These problems may somewhat limit comparison of our findings with other published studies.

We found that, in non-AIDS patients with pulmonary cryptococcosis, masses and air space consolidation are common ${ }^{4}$ and atelectasis, ${ }^{14}$ lymphadenopathy, ${ }^{4}$ and pleural effusion or empyema ${ }^{415}$ are relatively rare. Our findings are supported by some of the other series in the literature ${ }^{45}$ although not by Khoury et $a l^{\beta}$ who found no air space consolidation in nine immunocompetent patients.

We found a tendency for masses to occur in the right lung and lower lobes, but this was not statistically significant. In combination with other studies, ${ }^{410}$ this suggests that there is no significant lobar predilection in pulmonary cryptococcosis.

Abnormal chest radiographs were seen in $52 \%$ of patients with cryptococcal meningitis. This may be an early clue to the diagnosis and may have therapeutic implications, since resection of the pulmonary lesions may be indicated when antifungal therapy alone has proven inadequate. ${ }^{817}$ Two recent clinical series of immunocompetent patients with cryptococcal meningitis have shown a similar proportion with abnormal chest radiographs. ${ }^{18}{ }^{19}$

Twenty four $(80 \%)$ of 30 patients in whom variety typing was performed showed infection with $C$ neoformans var gattii. It has been postulated that this variety is more virulent than $C$ neoformans var neoformans, ${ }^{18}$ although the reasons why $C$ neoformans var gattii is rarely found in AIDS patients remain unclear. Furthermore, both pulmonary involvement in patients with cryptococcal meningitis and the presence of masses in those with pulmonary disease may be more common with $C$ neoformans var gattii. ${ }^{218}$ The clinical and radiological findings of this study may therefore not be directly comparable to other nonimmunocompromised groups in sub-tropical regions where $C$ neoformans var gattii is less common than $C$ neoformans var neoformans. ${ }^{20}$

In conclusion, cryptococcosis in HIV negative individuals can present with neurological, respiratory, or other symptoms or be an incidental finding at chest radiography. When the presentation is neurological the chest radiograph is abnormal in half of the cases, with one or more pulmonary masses being the most common finding. The radiographs of patients with respiratory symptoms usually show air space opacification ("cryptococcal pneumonia"), but may instead show an interstitial pattern or pulmonary mass(es). These features are different from those reported in patients with AIDS who are more likely to have interstitial opacities and lymphadenopathy.

We thank Drs DJ Lo, DM Howard, JN Burrow, S Selva Nayagam, MA Keen, and DF Madigan for caring for the patients included in this study.

1 Vanbreuseghem R, Takashio M. An atypical strain of Cryptococcus neoformans (San Felice) Vuillemin 1894. Part II: Cryptococcus neoformans var gattii var nov. Ann Soc Belge Cryptococcus neoformans var
Med Trop 1970;50:695-702.

2 Speed B, Dunt D. Clinical and host differences between infections with the two varieties of Cryptococcus neoformans. infections with the two varieties
Clin Infect Dis 1995;21:28-34.

3 Khoury MB, Godwin JD, Ravin CE, et al. Thoracic cryptococcosis: immunologic competence and radiologic appearance. $A \mathscr{F} R$ 1984;142:893-6.

4 Gordonson J, Birnbaum W, Jacobson G, et al. Pulmonary cryptococcosis. Radiology 1974;:112:557-61.

5 Cheong WY, Thomas A, Tan KP. Pulmonary cryptococcosis. Singapore Med f 1988;29:223-31.

6 Patz EF Jr, Goodman PC. Pulmonary cryptococcosis. $\mathcal{f}$ Thorac Imaging 1992;7:51-5.

7 Miller WT Jr, Edelman JM, Miller WT. Cryptococcal pulmonary infection in patients with AIDS: radiographic appearance. Radiology 1990;175:725-8.

8 Fisher D, Lo D, Burrow J, et al. Cryptococcus neoformans in tropical northern Australia: predominantly variant gattii tropical northern Australia: predominantly variant gatti
with good outcomes. Aust NZ F Med 1993;23:678-82.

9 Tuddenham WJ. Glossary of terms for thoracic radiology: recommendations of the nomenclature committee of the Fleischner society. $A f R \quad 1984 ; 143: 509-17$.

10 Feigin DS. Pulmonary cryptococcosis: radiologicpathologic correlates of its three forms. AfR 1983;141: 1263-72

11 Meyohas MC, Roux P, Bollens D, et al. Pulmonary cryptococcosis: localized and disseminated infections in 27 patients with AIDS. Clin Infect Dis 1995;21:628-33.

12 Friedman EP, Miller RF, Severn A, et al. Cryptococcal pneumonia in patients with the acquired immunodeficiency syndrome. Clin Radiol 1995;50:756-60.

13 Fraser RG, Paré JAP, Paré PD, et al. Diagnosis of diseases of the chest. 3rd ed. Philadelphia: Saunders, 1988: 459.

14 Carter EA, Henderson DW, McBride J, et al. Case report: complete lung collapse-an unusual presentation of cryptococcosis. Clin Radiol 1992;46:292-4.

15 Baird RW, Garfield AS. Cavitation and rupture of a pulmonary cryptococcoma in an immunocompetent man. $A m \mathcal{F}$ nary cryptococcoma in
Med 1994;97:309-11.

16 Smilack JD, Bellet RE, Talman WT Jr. Cryptococcal pleural effusion. FAMA 1975;232:639-41. 
17 Majid AA. Surgical resection of pulmonary cryptococcomas in the presence of cryptococcal meningitis. $\mathcal{F} R$ Coll Surg

18 Mitchell DH, Sorrell TC, Allworth AM, et al. Cryptococcal disease of the CNS in immunocompetent hosts: influence of cryptococcal variety on clinical manifestations and outcome. Clin Infect Dis 1995;20:611-6.
19 Lalloo D, Fisher D, Naraqi S, et al. Cryptococcal meningitis (C. neoformans var. gattii) leading to blindness in previously healthy melanesian adults in Papua New Guinea. $Q \mathcal{F} \mathrm{Med}$ 1994;87:343-9.

20 Kwon-Chung KJ, Bennett JE. Epidemiologic differences between the two varieties of Cryptococcus neoformans. Am $\mathcal{F}$ Epidemiol 1984;120:123-30. 